\title{
Avaliação microbiológica de maioneses artesanais fornecidas em serviços de alimentação do centro de Campina Grande - PB
}

\author{
Microbiological evaluation of artisanal mayonnaise provided in food services in the center of \\ Campina Grande - PB \\ Evaluación microbiológica de mayonesas artesanales prestadas em servicios alimentarios en el
} centro de Campina Grande - PB

\author{
Ana Clara Bezerra Felinto \\ ORCID: https://orcid.org/0000-0002-5611-6948 \\ Centro Universitário UniFacisa, Brasil \\ E-mail: anaclarabfelinto@gmail.com \\ Steffany Albuquerque do Bú \\ ORCID: https://orcid.org/0000-0002-4804-4657 \\ Centro Universitário UniFacisa, Brasil \\ E-mail: steffanyalbuquerque12@gmail.com \\ Eduarda Josefa Alves Marçal \\ ORCID: https://orcid.org/0000-0003-0021-9144 \\ Centro Universitário UniFacisa, Brasil \\ E-mail: eduardamarca1826@gmail.com \\ Igor Macêdo de Oliveira \\ ORCID: https://orcid.org/0000-0003-3717-7291 \\ Centro Universitário UniFacisa, Brasil \\ E-mail: Igormacedo1955@gmail.com \\ Joyce Almeida Lima \\ ORCID: https://orcid.org/0000-0002-5113-1903 \\ Centro Universitário UniFacisa, Brasil \\ E-mail: joycealmeida95@outlook.com \\ Juliana Barbosa de Sousa \\ ORCID: https://orcid.org/0000-0002-2228-8004 \\ Centro Universitário UniFacisa, Brasil \\ E-mail: juliana.bs628@gmail.com \\ Wagner Gomes de Melo \\ ORCID: https://orcid.org/0000-0001-5198-0457 \\ Centro Universitário UniFacisa, Brasil \\ E-mail: wagnerlegionario@hotmail.com \\ Mayra da Silva Cavalcanti \\ ORCID: https://orcid.org/0000-0003-1269-5324 \\ Centro Universitário UniFacisa, Brasil \\ E-mail: mayra_cavalcanti@yahoo.com.br
}

\begin{abstract}
Resumo
Com consumo elevado em lanchonetes da cidade a maionese é um produto feito à base de óleo, água e ovos; utilizada para realçar o sabor dos alimentos, podendo ser encontrada em diversas formas para uso. Em Campina Grande, muito se tem empregado a maionese de forma artesanal com adição de temperos naturais como forma de atrair ainda mais o paladar da população, o que torna esta prática uma fonte propícia para a contaminação do produto devido ao processo de manipulação. Com o objetivo de avaliar e quantificar a qualidade microbiológica deste insumo disponibilizado por lanchonete da cidade, o presente estudo foi traçado. Foram realizadas análises de acordo com os parâmetros regidos pela Normativa $n^{\circ} 12 / 01$, que aprova regulamento técnico sobre padrões microbiológicos em alimentos, visando quantificar Coliforme/g $\left(30^{\circ} \mathrm{C}\right)$, Salmonela spp. $/ 25 \mathrm{~g}$ e Estafilococos Coag. Pos/g. Foi averiguado que todas as amostrasde maionese pesquisadas todas elas se encontravam fora de algum dos parâmetros recomendados pela legislação, sendo o coliforme o microrganismo mais presente. Esta contaminação é indicativo de falta de cuidado na manipulação. Assim, este estudo mostra a importância de uma análise microbiológica para o controle, qualidade e segurança no preparo das maioneses artesanais, bem como a importância de boas práticas de fabricação para o alcance da segurança alimentar.
\end{abstract}

Palavras-chave: Qualidade; Boas práticas de fabricação; Microbiologia de alimentos. 


\begin{abstract}
With high consumption in snack bars in the city, mayonnaise is a product made from oil, water, and eggs; used to enhance the taste of food and can be found in various forms for use. In Campina Grande, a artisanal mayonnaise has been used with the addition of natural spices as a way to further attract the population's palate, which makes this practice a propitious source for contamination of the product due to the handling process. With the objective of evaluating and quantifying the microbiological quality of this input made available by a snack bar in the city, the present study was designed. Analyzes were carried out according to the parameters governed by Normative $\mathrm{n}^{\circ} 12 / 01$, which approves technical regulation on microbiological standards in food, aiming to quantify Coliform / g $\left(30^{\circ} \mathrm{C}\right)$, Salmonela spp. $/ 25 \mathrm{~g}$ and Staphylococcus Coag. Pos $/ \mathrm{g}$. It was verified that all the samples of mayonnaise researched were outside one of the parameters recommended by the legislation, with the coliform being the most present microorganism. This contamination is indicative of the lack of care in handling. Thus, this study shows the importance of a microbiological analysis for the control, quality and safety in the preparation of artisanal mayonnaise, as well as the importance of good manufacturing practices to achieve food security.
\end{abstract}

Keywords: Quality; Good manufacturing practices; Food microbiology.

\title{
Resumen
}

Con alto consumoen los cafetaría de la ciudad, la mayonesa es un product elaborado a base de aceite, agua y huevos; se utiliza para mejorar el sabor de los alimentos y se puede encontraren varias formas de uso. En Campina Grande se ha utilizado mucha mayonesa artesanal con el agregado de especias naturales como una forma de atraeraúnmás el paladar de la población, lo que hace de estapráctica una fuentepropicia para la contaminación del producto por el proceso de manipulación. Con el fin de evaluar y cuantificar la calidad microbiológica de este insumo que pone a disposición un snack bar de la ciudad, se diseñó el presente estudio. Los análisis se realizaron de acuerdo con los parámetros regidos por la Normativa $n^{\circ} 12 / 01$, que aprueba el reglamento técnico sobre estándares microbiológicos en alimentos, con el objetivo de cuantificar Coliformes / g (30 $\left.{ }^{\circ} \mathrm{C}\right)$, Salmonela spp./25g y Staphylococcus Coag. Pos / g. Se verificó que todas las muestras de mayonesa investigadas todas se encontra banfuera de alguno de los parámetros recomendados por la legislación, siendo el coliforme el microorganism más presente. Esta contaminación es indicativa de una falta de cuidado en la manipulación. Así, este estudiomuestra la importancia de un análisis microbiológico para el control, calidad e ino cuidaden la elaboración de mayonesa artesanal, así como la importancia de las buenas prácticas de fabricación para lograr la seguridad alimentaria.

Palabras clave: Calidad; Buenas prácticas de fabricación; Microbiología alimentaria.

\section{Introdução}

Nos últimos anos, a alimentação tem sido alvo constante de preocupação a nível mundial. As dificuldades relacionadas com o desenvolvimento produtivo de alimentos e sua excelência para o consumo ficaram mais evidentes devido ao avanço da globalização. E com isso, temos diversas causas que apontam o número progressivo de contaminação dos produtos alimentícios. Sintomas clínicos repentinos e associados ao trato digestivo como náuseas, vômitos e diarréia, são os mais constantes relacionados à intoxicação alimentar; mas dor abdominal e alterações neurológicas podem também estar presentes. O grau das manifestações clínicas vai alternar de acordo com o nível da infecção, a virulência do agente e a competência imunológica do hospedeiro (Neves,2016).

Existem cerca de 250 tipos de Doenças Transmitidas por Alimentos (DTA’s) e, dentre elas, muitas são oriundas de microrganismos patogênicos, que são responsáveis por diversos problemas de saúde pública e grandes perdas econômicas. A maioria dos surtos tem sido referente à ingestão de alimentos com boa aparência, sabor e odor normal, sem qualquer alteração sensorial visível. Isso acontece devido à dose infectante de patógenos alimentares que geralmente é maior que a quantidade de microrganismos fundamentais para degradar os alimentos (Oliveira et al., 2010).

Dados fornecidos pelo Ministério da Saúde apontam que a maionese é uma das principais responsáveis por surtos de doenças transmitidas por alimentos nos anos de 2007 e junho de 2016. Os alimentos obtidos através de ovos como a maionese estiveram presentes em 3,6\% dos casos de infecções alimentares relatadas no país (Faria,2016).

De acordo com a Resolução de Diretoria Colegiada (RDC) nº 276, de 22 de setembro de 2005 da Anvisa, a maionese é um produto cremoso em forma de emulsão estável, óleo em água, preparado a partir de óleo(s) vegetal (is), água e ovos podendo ser adicionado de outros ingredientes desde que não descaracterizem o produto, devendo este ser acidificado.Com a finalidade 
de realçar o sabor das maioneses, especiarias e ervas vem sendo utilizadas como forma de deixar o alimento mais palatável, conquistando a aceitação do público. Dentre as especiarias e ervas mais utilizada está o alecrim, orégano, sálvia, entre outros (Salgado; Carrer \& Danieli, 2006).

Entre os principais microrganismos encontrados em alimentos, estão as bactérias do grupo Coliformes termotolerantes, StaphylococcusCoagulase positivo e Salmonella spp. Os coliformes termotolerantes são bastonetes gram-negativos pertencentes a família Enterobacteriaceae com capacidade de fermentar lactose e produzir ácido e gás a $44{ }^{\circ} \mathrm{C}$ por 24 horas. Já o Staphylococcuscoagulase positivo trata-se de um grupo de cocos gram positivos, anaeróbios facultativos, onde seu mecanismo está relacionado à capacidade de produzir toxinas que são responsáveis por ocasionarem vários casos de intoxicações. O gênero Salmonella spp. Apresenta-se como bacilos gram negativos, anaeróbios facultativos, que pertencem a microbiota de animais de sangue quente, o que justifica o fato de estarem isoladas em alimentos de origem animal, e seus derivados, conseqüentemente fazendo desse grupo o principal agente causador de surtos de DTA no Brasil (Maas et al.,2020).

É de suma importância avaliar os surtos de doenças transmitidas por alimentos com o propósito de no decorrer das informações coletadas, seja possível um fornecimento de dados para a criação de métodos mais eficazes visando desta forma reduzir o número de ocorrências de surtos que podem levar, dependendo do estado do indivíduo acometido, até a morte (Ferraz et al.,2015).

Diante do que foi mencionado, o presente estudo teve como finalidade avaliar a qualidade microbiológica de maioneses artesanais fornecidas por estabelecimentos comerciais do centro da cidade de Campina Grande - PB, traçando um perfil e um possível alerta ao recorrente consumo deste tempero em lanchonetes da cidade.

\section{Metodologia}

As amostras de maionese foram adquiridas na cidade de Campina Grande - PB, diretamente com os fabricantes locais e em estabelecimentos comerciais, estes estabelecimentos eram cadastrados na prefeitura e a escolha das lanchonetes para a realizaçãoda análise foi devido ao estabelecimento fabricar de forma artenal a maionese para o consumo. Logo após as maionseses adquiridas foram levadas até o local de análise.

As seis amostras de maionese foram coletadas a partir da retirada de frações do local com cerca de 100g, depois acondicionadas em sacos estéreis e transportadas em caixa isotérmica com gelo e identificação. Estas foram mantidas sob refrigeração até o momento da análise.Os ensaios microbiológicos foram efetivados nos laboratórios de Microbiologia e Imunologia do Centro Universitário da Unifacisa.

As análises microbiológicas seguiram o protocolo da Resolução de Diretoria Colegiada (RDC) 12, de 02 de janeiro de 2001 e da instrução normativa 81, de 19 de dezembro de 2018. Que recomenda as análises de Coliforme/g (30 $\left.{ }^{\circ} \mathrm{C}\right)$, Salmonela spp./25g e Estafilococos Coag. Pos/gpara maioneses (Brasil, 2001; Brasil,2018).

O método de análise utilizado para contagem de coliformes foi o proposto por Silva et al. (2017) para contagem de coliformes totais em água e alimentos. Para tanto, foi aplicada a técnica dos tubos múltiplos com preparação de diluições até a $10^{-3}$. A amostra passou por diluição seriada e homogeneização por agitação em água peptonada e inoculação em Caldo Lactosado Bile Verde Brilhante (CLBVB), que permite a fermentação do grupo de coliformes. Após a inoculação, os tubos em triplicata foram levados para estufa à $35^{\circ} \mathrm{C}\left( \pm 2^{\circ} \mathrm{C}\right)$ por 48 horas $( \pm 2)$. Sendo a positividade dada pela presençadogáspresono tubodeDurham.

Para detectar Staphylococcus foi seguida a metodologia descrita por Resta \& Oliveira (2013). Para tanto, uma alíquota de 2,5g de cada amostra do alimento foram pesadas assepticamente e homogeneizadas com 22,5ml de água peptonada $0,1 \%$. Depois de um período de 30 a 60 minutos sob refrigeração, as amostras foram submetidas à homogeneização. Diluições 
decimais, a partir da diluição $10^{-1}$, foram preparadas em tubos contendo $9,0 \mathrm{ml}$ de água peptonada $0,1 \%$ estéril, até a diluição de $10^{-3}$. Alíquotas de $0,1 \mathrm{ml}$ das diluições foram semeadas em ágar Vogel e Johnson em duplicata. As placas foram incubadas a $35^{\circ} \mathrm{C}( \pm 2)$. e, após 48 horas, realizadas a identificação e a contagem das colônias que apresentavam as características típicas de Staphylococcus spp.

Para a determinação de presença de Salmonella foi utilizado o método descrito por Silva et al. (2017) para presença ou ausência em alimentos. Esses métodos são aplicados em todos os alimentos de consumo humano. Para tanto, a amostra foi diluída em água peptonada para pré-enriquecimento; e deixada em repouso à $35^{\circ} \mathrm{C}\left( \pm 1^{\circ}\right)$ por 18 horas $( \pm 2)$. Depois foi transferido $1 \mathrm{ml}$ do conteúdo para o caldo de Tetrionato e incubada à $37^{\circ} \mathrm{C}\left( \pm 1^{\circ}\right)$ por 24 horas $( \pm 3)$. Após esse período, foi inoculado em meio de Ágar Entérico de Hectoen (HE) e Ágar Bismuto Sulfito (BS), num volume de $0,1 \mathrm{ml}$ e novamente incubada à $37^{\circ} \mathrm{C}$ $\left( \pm 1^{\circ}\right)$ por 24horas $( \pm 3)$. Esta análise foi feita em duplicata, por esgotamento (Figura 2). As colônias típicas no Ágar HE eram transparentes, verde-azuladas, com ou sem centro preto, e as no Ágar BS eram colônias castanhas, cinza ou pretas, com ou sem brilho metálico.

\section{Resultados e discussão}

Os resultados encontrados nas análises estão demonstrados na Tabela 1. Das maioneses analisadas todas apresentaram valores acima do preconizado pela $\mathrm{RDC} \mathrm{n}{ }^{\circ} 12$, de 02 de Janeiro de 2001 para análise de coliformes totais à $35^{\circ} \mathrm{C}$ (Brasil, 2001) .

Tabela 1 - Avaliação da qualidade higiênica sanitária das maioneses temperadas.

\begin{tabular}{cccc}
\hline & \multicolumn{3}{c}{ Análises } \\
\cline { 2 - 4 } Maionese & Salmonella $($ UFC/g) & $\begin{array}{c}\text { Staphylococcusaureus } \\
\text { (UFC/g) }\end{array}$ & $\begin{array}{c}\text { Coliformes totais à } \\
35^{\circ} \mathrm{C}(\mathrm{NMP} / \mathrm{g})\end{array}$ \\
\hline M1 & Aresente & Ausente & $1,1 \times 10^{3}$ \\
M3 & Ausente & Ausente & $1,5 \times 10^{1}$ \\
M4 & Ausente & Ausente & $2,3 \times 10^{1}$ \\
M5 & Presente & Ausente & $4,3 \times 10^{1}$ \\
M6 & Ausente & Ausente & $4,6 \times 10^{2}$ \\
Legislação* & Ausente & Ausente & $1,5 \times 10^{2}$ \\
\hline
\end{tabular}

*Segundo a Resolução Normativa ${ }^{\circ} 12 / 01$ que aprovar regulamento técnico sobre padrões microbiológicos para alimentos.

Fonte: Autores (2021).

Casemiro e Martins (2015) em seu estudo avaliaram a prevalência da contaminação microbiológica em maioneses caseiras comercializadas em carrinhos de cachorro-quente e em todas suas amostras analisadas havia a presença de coliformes fecais. Silva et al. (2015) encontraram resultados significativos do que o presente estudo ao analisar maioneses comercializadas em Pombal - PB. Foram realizadas análises de três amostras de maionese, ao final da pesquisa as amostras apresentaram pequenos níveis de coliformes, o que não indica necessariamente contaminação fecal recente, mas talvez manipulação em condições precárias de higiene, ou manejo inadequado por parte dos manipuladores.

No município de Texeira de Freitas na Bahia, os pesquisadores Fernandes, Gonçalves e Fortuna (2015) confirmaram a 
presença de coliformes totais em 60\% das amostras comercializadas em lanchonetes. Faria (2016) analisou amostras de maioneses caseiras servidas em lanchonetes do Municipío de Dores do Indaiá - MG e verificou que nas amostras analisadas não houve a detecção de coliformes. Já em uma pesquisa realizada em restaurantes do tipoself-service localizados no Rio de Janeiro, demonstraram que $100 \%$ de suas amostras analisadas das (saladas de maionese com ovos) observou-se a presença decoliformes a $35^{\circ} \mathrm{C}$, sendo este um indicativo de contaminação. (Bricio; Leite \& Viana, 2005).

Para Salmonella sp. foi possível ver a detecção em apenas duas amostras das analisadas, M1 e M4. Nadvorny, Figueiredo \& Schmidt(2000), em sua pesquisa realizada no Rio Grande do Sul relataram que dos 99 surtos de DTA's ocorridos em sua cidade, $74,7 \%$, foram ocasionados por Samonella sp., dentre os quais alimentos preparados com ovosestiveram envolvidos em 72,2 dos surtos de salmonellose.

A Salmonella spp. é um microrganismo entérico responsável por diversas intoxicações alimentares, sendo um dos principais agentes envolvidos em surtos ocasionados em vários países. A sua presença em alimentos é um indicador relevante de saúde pública. Deve-se ressaltar que a maioria dos sorotipos desse gênero são patogênicos ao homem, apresentando diferenças de sintomatologia em decorrência da variação no mecanismo de infecção, além da idade e da resposta imune do hospedeiro (Shinohara et al., 2008).

As infecções alimentares ocasionadas por este patógeno são causadas pela ingestão de produtos contaminados com este microrganismo. A contaminação pode ocorrer por duas formas: alimentos de origem animal infectados; ou por meio de contaminação cruzada, que consiste na transmissão de contaminante do alimento por meio de equipamentos, manipuladores, roedores, insetos ou até mesmo de outros alimentos, sendo este um fator presente nos surtos de salmonelose em restaurantes e lanchonetes (Domingos, 2015).

Os sintomas decorrentes deste microrganismo incluem cólicas abdominais, náuseas, vômitos, diarréias, calafrios e febre. São vulneráveis à infecção por este, qualquer indivíduo que consuma alimentos insuficientemente cozidos ou crus, feitos à base de carnes ou ovos, já que estes contaminantes são amplamente encontrados na natureza, sendo o trato gastrointestinal do homem e dos animais o principal reservatório natural (Cardoso \& Carvalho, 2006).

Já para Staphylococcus aureusnão houve crescimento em nenhuma das amostras analisadas, isso significa que todas as amostram se encontram dentro do preconizado pela Legislação vigente. No Município do Rio Grande do Norte - CE, uma pesquisa realizada por Laranjeira et al. (2020) com maioneses caseiras, o microrganismo que mais apresentou incidência foi o Staphylococcus aureusem $89,6 \%$ das amostras analisadas, os resultados obtidos nessa pesquisa podem estar relacionados com oarmazenamento e condições de higiene inadequadas.

A bactéria Staphylococcus aureus está associada com a ausência de treinamentos dos manipuladores, que é uma técnica indispensável para orientar e educar os manipuladores quanto aos cuidados essenciais na conservação, manipulação e consumo dos alimentos. A ausência destes conhecimentos leva ao manuseio inadequado, uma vez que manipuladores não possuem capacidade na execução e conhecimento em relação às boas práticas de segurança alimentar, podendo maximizar o número de surto de DTA (Araújo, 2019).

O avanço econômico e a busca por se enquadrar no contexto atual trouxeram mudanças na qualidade alimentar da comunidade. Esta flutuação compreende o aumento da procura pelos serviços de alimentação, os quais atendem o desejo dos consumidores que buscam refeições adequadas no que se refere à praticidade de aquisição e preparo ou consumo fora do lar. Adepto a este fator sabe-se que o desempenho dos serviços de alimentação em larga escala é, sobretudo perigoso em função da forma em que os alimentos são armazenados e manipulados (Silva et al., 2017).

A produção de maioneses artesanais no centro de Campina Grande é uma prática inovadora vinda dos estabelecimentos oriundas de lanchonetes e fast-foods como uma forma de incrementar ainda mais sabor nos lanches ofertado, além disso, é uma 
forma de baratear o custo do fornecimento, tendo em vista que para o preparo de maioneses utilizam-se poucos ingredientes e o rendimento é considerável.

Todavia, durante a coleta das amostras nos diversos pontos comerciais de intensa movimentação do centro de Campina Grande, foi perceptível a ausência das Boas Práticas de Manipulação (BPM) durante o manuseio do alimento. Os manipuladores não realizavam a utilização de Equipamentos de Proteção Individual (EPI`s) que são apontados como indispensáveis. Entre estes estão em destaque: touca, luva, máscara e avental. Foi possível acompanhar que em algumas das lanchonetes do estudo o funcionário que atendia no caixa era a mesma pessoa que fazia o preparo do alimento.

De acordo com a Resolução $\mathrm{N}^{\circ}$ 216, de 15 de Setembro de 2004: 'Os manipuladores não devem fumar, falar desnecessariamente, cantar, assobiar, espirrar, cuspir, tossir, comer, manipular dinheiro ou praticar outros atos que possam contaminar o alimento, durante o desempenho das atividades" (Brasil, 2004).

Segundo Marchi et al. (2011), no Brasil, entre os anos de 1999 e 2008, foram notificados 6.062 surtos de DTA. O ano com maior número de notificações foi em 2005 (n=923), em seguida 2001 (n= 897) e 2002 (n=823). Na mesma época, Santa Catarina registrou 530 surtos. No Rio Grande do Sul, ocorreram 99 surtos de DTA no ano 2000, e no período entre 1999 e 2004, os Estados do Rio Grande do Sul, São Paulo e Paraná lideraram o número de notificações de surtos de DTA, com 928, 663 e 625 registros, respectivamente, resultado de uma notificação com mais eficácia.

No decorrer das coletas de maioneses artesanais no centro de Campina Grande foi notório perceber a falta de conscientização dos funcionários quanto à manipulação dos alimentos. As maioneses eram acopladas em sacos plásticos pequenos enrolados ou até mesmo em copos descartáveis cobertos com filme plástico. Se o consumo dos alimentos fosse na lanchonete em alguns locais a maionese estava pronta e exposta a céu aberto dentro de um pote de margarina de $3 \mathrm{~kg}$, facilitando desta forma a proliferação de bactérias.

Diante destas circunstâncias é possível afirmar que a ausência da Boa Prática de Manipulação e a falta de higiene dos manipuladores pode deixar o alimento mais susceptível ao risco de contaminação colocando neste caso a saúde da população em risco, sem que haja um controle de qualidade do serviço ofertado, contribuindo dessa forma para o aumento de índices de surtos originados pela DTA.

\section{Conclusão}

A partir das análises microbiológicas foi possível concluir que as maioneses artesanais fornecidas em serviços de alimentação do centro de Campina Grande - PB encontram-se impróprias para o consumo da população, tendo em vista que os microrganismos analisados se encontram fora dos parâmetros em que rege a legislação. A ausência de boas práticas de manipulação e fabricação podem ter sido um fator importante para a contaminação das amostras.

Sendo assim, este trabalho apresenta imensa relevância na demonstração dos seus resultados para o risco no consumo destes alimentos e indicando a necessidade de treinamento de toda a equipe presente nestes estabelecimentos. Desta forma ressalta-se a importância do nutricionista ao agrega valor ao estabelecimento por conhecer as BPM e poder montar um fluxo de produção, conservação e distribuição, além de verificar se as normas exigidas pela vigilância sanitária estão sendo realizada, este profissional pode verificar a qualidade dos alimentos ofertados, a higienização do alimento e a organização do ambiente em que trabalha. Mais estudos como este são necessários a fim de conscientizar a importância de um serviço que segue todas as normas de boas práticas e prezam pela saúde da população ao qual serve.

Entendemos da necessidade de um aprofundamento maior sobre o tema que abarque uma maior quantidade de amostras das maioneses artesanais no âmbito municipal, a fim de ter um esclarecimento maior sobre o tema o tema abordado. 


\section{Referências}

Araújo, T. Da S. (2019). Análise microbiológica de molhos caseiros comercializados em food trucks e restaurantes do munícipio de Bebedouro-SP. Revista Ciências Nutricionais, 3, 14-19.

Brasil. Agência Nacional de Vigilância Sanitária- ANVISA. Resolução - RDC No 216, de 2006.

Brasil. 15 de Setembro de 2004. Estabelece procedimentos de boas Práticas para serviço de alimentação, garantindo as condições higiênico-sanitárias do alimento preparado. Diário Oficial da União, Brasília, DF, 17 setembro de 2004.

Brasil. Anvisa - Agência Nacional de Vigilância Sanitária. Anvisa - Agência Nacional de Vigilância Sanitária. Resolução RDC nº 12 , de 02 de setembro de 2001: Aprova o "Regulamento Técnico Sobre Padrões Microbiológicos Para Alimentos". 2001.

Bricio, S.M.L., Leite, S.G. F. \& Viana, C (2005). Avaliação microbiológica de salpicão de frango e salada de maionese com ovos servidos em restaurantes selfservice na cidade do Rio de Janeiro. Higiene alimentar, 90-95

Cardoso, T. G. \& Carvalho, V. M (2006). Toxinfecção alimentar por Salmonella spp Food bornedisea secausedby Salmonella spp. Revista do Instituto de Ciência e Saúde, v. 24, n. 2, p. 95-101.

Casemiro, L.P. \& Martins, A. L. O (2016). Prevalência de contaminação microbiológica e parasitológica de maioneses caseiras comercializadas em carrinhos de cachorro- quente. Revista Brasileira de Análises Clínicas, 48, 394-9.

Domingos, I., Brunelli, S. R. \& Baldotto, S. B (2015). 'Salmonella spp'’- Uma revisão.Revista FAIT, Itapeva, $2,15$.

Faria, J. N. M (2016). Análise microbiológica de maionese caseira servidas em lanchonetes nacidade de Dores do Indaía-MG. Tese de Doutorado, Minas Gerais.

Fernandes, A. M., Kern, M. G. \& Fortuna, J. L (2015).'Pesquisa de coliformes e estafilococos em maioneses artesanais de lanchonetes do município de Texeira de Freitas: BA. "'Revista Higiene. Alimentar,' 200-205

Ferraz, R. R. N., Santana, F. T., Barnabé, A. S. \& Fornari, J. V (2015). Investigação desurtos de doenças transmitidas por alimentos como ferramenta de gestão em saúde de unidades de alimentação e nutrição. RACI, Getúlio Vargas, 9(19)

Laranjeira, F. D. L., Monteiro, M. D. F. G., Saraiva, C. R. N., Aquino, P. E. A., Lima, M. M., De Souza Júnior, D. L. \& Leandro, L. M (2020). G.Pesquisa De Escherichia coli, Salmonella sp. e Staphylococcus aureus em maioneses caseiras comercializadas no município de Juazeiro do Norte-CE. Revista Interfaces: Saúde, Humanas e Tecnologia, 8, 554-560.

Maas, A., Valiatti, T. B., Rosa, N. B., Leite, P. F., Romão, N. F. \& Sobral, F. D.O. S (2020). Avaliação microbiológica de maionese caseira comercializada no município de Ji- Paraná, Rondônia. Ciência e Desenvolvimento -Revista eletrônica da FAINOR, 13, 205-214.

Malacrida, A. M., Dias, V. H. C. \& Lima, C. L (2017). "Perfil epidemiológico das doenças bacterianas transmitidas por alimentos no Brasil." Revista de Ciência Veterinária e Saúde Pública, 4, 158-162.

Marchi, D. M., Baggio, N., Teo, C. R. P. A. \& Busato, M. A (2011). Ocorrência de surtos dedoenças transmitidas por alimentos no Município de Chapecó, Estado de Santa Catarina, Brasil, no período de 1995 a 2007. Epidemiologia e serviços de saúde, 20, 401-407.

Nadvorny, A., Figueiredo, D. M. S. \& Schmidt, V (2004). Ocorrência de Salmonella sp. em surtos de doenças transmitidas por alimentos no Rio Grande do Sul em 2000. Acta Scientiae Veterinariae, 32, 47-51.

Neves, M. C. D. M (2016). Levantamento de dados oriundos do DATASUS relativo à ocorrências / surtos de intoxicação alimentar no Brasil de $2007-2014$. João Pessoa.

Oliveira, A. B. A. D., Paula, C. M. D. D., Capalonga, R., Cardoso, M. R. D. I. \& Tondo, E. C (2010). Doenças transmitidas por alimentos, principais agentes etiológicos e aspectos gerais: Uma Revisão. Revista Hcpa, 30, .279-285

Resta, M. S. A. \& De Oliveira, T. C. R. M (2013). Avaliação do padrão estafilococos coagulase positiva estabelecido pela legislação brasileira para massas alimentícias. Brazilian Journal of Food Technology, 16, 319-325

Salgado, J. M., Carrer, J. C. \& Danieli, F. (2006). Avaliação sensorial de maionese tradicional e maionese enriquecida com ervas aromáticas. Food Science and Technology. Campinas v. 26, n. 4, p. 731-734, out./dez.

Shinohara, N. K.S., Barros, V. B., Jimenez, S. M. C., Machado, E. C. L.,Dutra, R. A. F. \& Filho, J. L. L (2008). Samonella spp., importante agente patógeno veiculado em alimentos. Revista Ciências \& Saúde Coletiva, 13, 1675-1683.

Silva, G. B., Oliveira, R. P. D. S. \& Santos, M (2015). Análises Microbiológicas de Maionese de Banana Verde (Musa sapientum). IV Congresso Estadual de Iniciação Científica,

Silva, N., Junqueira, A. C. V., Silveira, A. F. N., Taniwaki, H. M., Gomes, R.A. R. \& Okazaki, M. M (2017). Manual de Métodos de Análises Microbiológica de Alimentos e Água. (5a ed.), Blucher.

Souza, E. L. D. \& Silva, C. A. D (2004). Qualidade sanitária de equipamentos, superfícies, água e mãos de manipuladores de alguns estabelecimentos que comercializam alimentos na cidade de João Pessoa, PB. Revista Higiene Alimentar, 98-102. 